\title{
Effect of Music Interventions on Sedation in Children Undergoing Magnetic Resonance Imaging: Clinical Trial
}

\author{
Ambika Mathur ${ }^{1,{ }^{*}}$, Aarti Kamat ${ }^{1}$, Blythe Philp ${ }^{2}$, Jennifer Tabb ${ }^{1}$, Ronald Thomas ${ }^{3}$, \\ Prashant Mahajan $^{3}$, John Caldwell ${ }^{1}$ and Deepak Kamat ${ }^{3}$ \\ ${ }^{1}$ Wayne State University, Detroit, MI, USA \\ ${ }^{2}$ Child Life Services, Children's Hospital of Michigan, Detroit, MI, USA \\ ${ }^{3}$ Department of Pediatrics, Wayne State University School of Medicine, Children's Hospital of Michigan, \\ Detroit, MI, USA
}

\begin{abstract}
Background: Although parenteral sedation is often required in MRI studies in children, it is stressful and increases the cost of healthcare.

Objectives: We evaluated the impact of music interventions in children receiving parenteral sedation for MRI studies on total number of doses of sedation medications, sedation time, levels of cortisol and cytokines, sedation success, adverse events, parental satisfaction, and cost savings.

Methods: We conducted a prospective open unblinded four-arm clinical evaluation of interventions on 471 children 1-12 years of age undergoing MRI and receiving parenteral sedation. Children were assigned to active music therapy (AMT), facilitated music listening (FML), and as comparison another intervention (child life intervention or CLI), or no intervention (NI); measures included number of doses of sedation medications, time of sedation, sedation success, adverse events, parental satisfaction, and salivary levels of the stress hormone cortisol and pro-inflammatory cytokines, before and after intervention.
\end{abstract}

Results: The total number of sedation doses, total sedation time, and levels of salivary cortisol and cytokines did not differ between the four groups. One FMLA choice, Wee Sing Animal Songs, resulted in significant decrease in total sedation time and reduction of associated costs.

Conclusions: The use of one type of FML led to decreased total sedation time in children. This is an important finding since FML is an inexpensive non-invasive intervention which could be of significant time and cost saving benefits.

Keywords: Active Music Therapy, Facilitated Music Listening, MRI, Cytokines.

\section{INTRODUCTION}

Magnetic resonance imaging (MRI) is used in the evaluation and management of several pediatric illnesses. Sedation is often required to improve image quality, reduce motion artifact and alleviate anxiety in children undergoing MRI [1]. However, sedation is problematic, because it is associated with adverse events such as desaturation requiring airway maneuvers and oxygen supplementation [2-5]. It is therefore important to identify non-invasive soothing and relaxing ways to mitigate the effects of sedation, especially in children.

Music has been used for centuries by every culture to soothe and relax the mind, making the use of music to alleviate stressful conditions very natural $[6,7]$. As defined by Ghetti [8], Music Therapy for procedural support is "the use of music and aspects of the therapeutic relationship to promote healthy coping and

*Address correspondence to this author at the Graduate School, Wayne State University, 5057 Woodward Ave., 6304.1, Detroit, MI 48202, USA;

Tel: 313-577-2172; Fax: 313-577-2903; E-mail: ambika.mathur@wayne.edu decrease distress in individuals undergoing medical procedures." In this theoretical model, the music therapist considers a variety of experience moderators including personal and contextual variables of the patient, family and medical procedure, and facilitates patient and family involvement in an individualized music intervention in which the role of the therapist, the music and the responses of the patient and family all serve to evaluate the patient's experience of the medical procedure. Outcomes such as patient's perception of pain, use of coping strategies and adaptive or maladaptive behaviors are assessed within the moment and used to inform any alterations in the music therapy intervention. The process is cyclical and continues throughout the course of the medical procedure.

Music interventions in pediatric procedural support have frequently been classified as "active" or "passive" [9]. For purposes of the present study, Active Music Therapy (AMT) is defined as an intervention in which a board-certified music therapist interacts with patients directly, involving patients in an interpersonal music experience through playing and singing. Active music 
interventions for medical procedural support most commonly fall into two classifications. Music Alternate Engagement that are therapeutic music activities which provides both an alternate focus and supports the patient's need to attend to the procedure at times. Music Assisted Relaxation which uses elements of music such as tempo, complexity of melody, and timbre to structure the relaxation response, and Integration in which music is used to give a voice or auditory representation to patient experiences both negative i.e. pain, anxiety or positive i.e. mastery, calming responses [8, 10, 11]. Interventions most commonly involved in AMT include singing, musical play with simple or adapted instruments, and using patient response to inform and guide therapist provision of live music $[7,10,12]$.

Facilitated Music Listening (FML) is defined in this study as an intervention in which a music therapist helps a patient select appropriate recorded music and trains the patient and their family to use recorded music to support positive coping. Such therapies have been shown to alleviate stress and anxiety in a number of settings in children and adults [7,12, 13-17]. A metaanalysis by Standley \& Whipple [18] indicated positive clinical results when recorded music was applied as a form of medical procedural support, and indicated no significant difference based on the individual selecting the music: the patient, therapist or medical staff. The study did indicate greater results when patients were active participants, as such, incorporating patients in the selection of the music provided is considered best practice. Recorded music selected and adapted by a music therapist has also been shown to increase parental participation and competence in medical care of children, which can also contribute to greater compliance and patient coping [19]. Music Therapy (MT) impacts physiologic responses including stress and immune systems [7, 13, 14]. MT has been shown to lower stress by lowering production of the stress hormone cortisol in groups of people experiencing high levels of stress such as students taking examinations, air control operators etc. [7, 13, 14]. Studies [14, 20] have shown the effect of MT on immune responses in several disorders with changes observed in cortisol and inflammatory biomarker levels such as cytokines Interleukin-1alpha (IL-1 $\alpha$ ), IL- $\beta$, IL-6, IL-8, Tumor Necrosis Factor (TNF) and Monocyte Chemotactic Protein-1 (MCP-1).

A systematic review of controlled trials on the effect of music for pain and anxiety in children undergoing medical procedures reviewed 19 (5 AMT, 14 FML) parallel randomized control trials and concluded that FML is as effective as AMT in significantly reducing pain and anxiety [16]. Further, MT is effective in reducing the amount of pharmacological interventions in a cost-effective manner [21]. In a study that compared the effect of AMT vs. the use of chloral hydrate to induce sedation in children undergoing EEG procedure, AMT had a significantly positive effect for level of sleep/sedation $(p<.001)$ and length of sleep/sedation $(p<.001)$ in comparison to the chloral hydrate group [22]. Another study for MT procedural support indicated success in eliminating patient sedation, reducing procedural time and in the number of medical professionals present during ECGs, CT scans, IV starts, X-rays, ventilator extubations, and EEGs [12]. These finding promote music not only as an effective and safe alternative to sedation medications but also as a cost saving measure. Although there have been studies that have compared the efficacy of individual therapies, to our knowledge, no prospective studies could be found which compared Music Therapy (AMT, FML) with traditional care for children undergoing sedation for MRI.

The main objective of our study was to evaluate the impact of music interventions (AMT, FML) compared to no intervention ( $\mathrm{NI}$ ) or another comparison child life intervention technique, Child Life Intervention (CLI) in children receiving parenteral sedation for MRI.

\section{METHODS}

\subsection{Study Design and Procedures}

We conducted a prospective four-arm clinical study on children 12 years of age and younger scheduled to receive parenteral sedation for MRI studies at Wayne State University/Children's Hospital of Michigan, Detroit, Michigan. All patients between the ages of 1-12 years scheduled for MRI study and who presented to our sedation suite were eligible. Exclusion criteria were: significant congenital abnormalities including central nervous system malformations and genetic syndromes. Patients were recruited between January 2010 and September 2011 by the research technicians as they arrived for the study; all patients/caregivers agreed to participate in the study. Following appropriate informed consent/assent, patients were assigned to one of four intervention arms: AMT, FML, CLI, or NI. Based on the work schedules of the Music Therapist and Child Life Specialist, we assigned specific days for allocation of patients to each intervention arm. To ensure balanced 
enrollment over time to the arms, we sometimes adjusted the allocations at random. For obvious reasons, the study could not be blinded. Importantly, since the study was conducted immediately following consent, we did not lose a single patient in the study i.e. $100 \%$ enrollment was achieved and all subjects were analyzed for primary outcomes.

Parent proximity to patient during IV start and sedation, soothing touch, and verbal reassurance were encouraged. If siblings or other child family members were present, they were included in interventions provided. Immediately following consent, patients and their caregivers were escorted to the intervention area. Saliva was collected for measuring stress hormone (cortisol) and the inflammatory cytokines (IL-1 $\alpha, \mathrm{IL}-\beta$, IL-6, IL-8, TNF and MCP-1) levels by swabbing the patients mouths with sorbettes (Salimetrics, Corning, NY) as recommended by the manufacturer. Saliva samples were centrifuged and stored at $-20^{\circ} \mathrm{C}$ until analysis. The MT-BC or CCLS performed the intervention for 10-15 minutes prior to the IV start. The research coordinator observed the start of the intravenous (IV) sedation and documented the participant's responses based on the CHEOPS (Children's Hospital of Eastern Ontario Pain Scale), a behavioral scale that has been used to evaluate postoperative pain in children. Sedative medications were administered according to institutional protocol. The MT-BC, or CCLS had continued presence with the child and family until the child was taken to the MRI scanner. The total sedation time (TST) was recorded from the time of start of the first sedation medication until the time the patient was discharged from the hospital. At the time of discharge, a second saliva sample was collected, caregivers completed the questionnaire, and were compensated for participating in the study.

As per our institutional protocol, children 1-7 years are initially sedated with pentobarbital $3 \mathrm{mg} / \mathrm{kg}$ intravenously. If the child fails to achieve an adequate level of sedation, additional doses of pentobarbital (up to a maximum of $10 \mathrm{mg} / \mathrm{kg})$ or fentanyl $(1-3 \mathrm{mcg} / \mathrm{kg})$ are administered. Children older than eight years are given midazolam IV (initial dose of $0.1-0.2 \mathrm{mg} / \mathrm{kg}$ with a maximum dose of $10 \mathrm{mg}$ ). All patients are monitored by a cardio respiratory monitor, pulse oximeter and an end tidal carbon dioxide monitor. Vital signs are obtained at baseline and every five minutes after administration of each medication and every 10 minutes until discharge.

\section{STUDY ARMS}

\subsection{AMT}

Participants were engaged in interactive music play by a Board Certified Music Therapist (MT-BC). AMT and FML were provided by the same MT-BC for all the patients. Prior to IV insertion for sedation, the MT-BC engaged the patient and family in developmentally appropriate interactive music play using small percussion instruments with MT-BC accompanying via guitar and singing. The MT-BC also introduced and encouraged selection and practice of active music techniques to promote compliance and positive coping during IV catheter insertion. These techniques included continued music play with decreased movement such as single hand keyboard play or guitar strumming, use of familiar songs to promote singing or vocalization, use of ocean drum to provide relaxing soundscape and alternate focus, and use of harmonica to promote deep breathing. Procedural support interventions employed during the IV catheter insertion were based on patient preference using both direct statements from the patient and family as well as observations of which instruments and interventions elicited the greatest level of patient engagement. Musical interventions provided during the IV catheter insertion were modified in the moment based on the demands of the procedure and the level of patient engagement. All available developmentally appropriate music therapy tools were presented to each participant (for example a harmonica would not have been provided to an 18 month old and the early childhood wave drum would not have been provided to a developmentally typical 8 year old) and play was conducted based on the child's preference. Additionally all developmentally appropriate procedural support techniques were presented to the child/parents and were applied during the IV insertion based on family preference. Procedural support techniques were those that were considered to be engaging to the patient while promoting limited movement and also included techniques to promote deep breathing. Harmonica was considered appropriate for this task as it could be played with one hand or positioned by the MT-BC and provided auditory feedback for both inhalation and exhalation which is key to encouraging deep breathing. This procedure occurred at the start of the IV sedation and not during the actual imaging procedure since deep breathing is an established procedural intervention for venipuncture. Musical tempo was entrained to the patient's respiratory rate and gradually decreased during sedation. Complexity 
of musical stimuli was also decreased during the course of sedation.

\subsection{FML}

The MT-BC presented a selection of $6 \mathrm{CDs}$ to patients and families. CDs were divided by developmental level and included a relaxing/sedative selection and a stimulating/engaging selection. Music selection was somewhat fixed by the researchers to decrease variance in the sound stimuli offered; however, patient preference was encouraged by offering a choice of 6 CDs. CDs were selected based on selections used frequently by the inpatient Music Therapy program at the hospital in which the study took

Table 1: Types of CDs Used for Facilitated Music Listening

\begin{tabular}{|c|c|c|c|}
\hline CD Citation & Age Range & Intended Purpose & Therapeutic Elements \\
\hline $\begin{array}{c}\text { Beale, P., Nipp, S. } \\
\text { [Various Artists]. Wee } \\
\text { Sing Animals Animals } \\
\text { Animals. New York: PSS. } \\
\text { (2006). }\end{array}$ & $1-3$ years & Engaging/Stimulative & $\begin{array}{l}\text {-Familiar songs reduce stress of unfamiliar } \\
\text { environment. } \\
\text {-Short duration creates increased presentation of novel } \\
\text { stimuli. } \\
\text {-Use of children's voices to encourage singing/play. } \\
\text {-Use of animal sounds to encourage vocalizing/play. } \\
\text {-Upbeat tempo, simple rhythmic structure promotes } \\
\text { clapping/movement. }\end{array}$ \\
\hline $\begin{array}{l}\text { Various Composers. } \\
\text { [Gunnel, M., Frias. K]. } \\
\text { Slumber Baby. Ann } \\
\text { Arbor, MI: Brookwood } \\
\text { Studios. (2005). }\end{array}$ & $1-3$ years & Relaxing/Sedative & $\begin{array}{l}\text {-Slow steady tempo promotes rhythmic soothing touch } \\
\text { provided by family, rocking feel } 6 / 8 \text { meter. } \\
\text {-Simple musical arrangement including solo voice and } \\
\text { accompanying instrument or instrumental duets. } \\
\text {-Short duration of songs creates increased } \\
\text { presentation of calm yet novel stimuli. } \\
\text {-Major and minor melodies included. }\end{array}$ \\
\hline $\begin{array}{l}\text { Various Composers. } \\
\text { [Various Artists]. Disney } \\
\text { Greatest Vol. 1. Walt } \\
\text { Disney Records. (2001). }\end{array}$ & $4-7$ years & Engaging/Stimulative & $\begin{array}{l}\text {-Familiar songs from popular movies creates coupling } \\
\text { of sound stimuli and creative play involving movie } \\
\text { character, themes, plot lines etc. } \\
\text {-Medium duration of songs promotes more focused } \\
\text { attention while still providing novel stimuli amongst } \\
\text { songs. } \\
\text {-Voices of familiar characters promotes singing. } \\
\text {-Upbeat simple rhythmic structure promotes } \\
\text { clapping/movement. } \\
\text {-Full orchestral arrangements promotes more complex } \\
\text { listening experience for engagement. }\end{array}$ \\
\hline $\begin{array}{l}\text { Various Composers. } \\
\text { [Various Artists]. The } \\
\text { Ultimate Most Relaxing } \\
\text { Classics for Kids. Dennon } \\
\text { Records (2007). }\end{array}$ & Ages 4-7 & Relaxing/Sedative & $\begin{array}{l}\text {-Small ensemble instrumentation creates clarity and } \\
\text { simplicity in sound. } \\
\text {-No major changes in volume level or tonality of music. } \\
\text {-Familiar classical pieces (used frequently in movies, } \\
\text { commercials etc). } \\
\text {-Slow tempo and limited dissonance or musical tension } \\
\text { to aid in relaxation. }\end{array}$ \\
\hline $\begin{array}{l}\text { Various Composers. } \\
\text { [Various Artists]. Radio } \\
\text { Disney Jams Vol. 11. Walt } \\
\text { Disney Records (2009). }\end{array}$ & Ages 8-12 & Engaging/Stimulative & $\begin{array}{l}\text {-Familiar songs frequently played on radio or in movies } \\
\text { to promote singing and song recognition. } \\
\text {-Upbeat tempo and major tonality to promote positive } \\
\text { upbeat attitude in patients. } \\
\text {-Popular instrumentation including keyboards, drums, } \\
\text { guitars, and electronic instruments. } \\
\text {-Moderate song length to promote extended attention } \\
\text { and provided regular novel stimuli. }\end{array}$ \\
\hline $\begin{array}{l}\text { Wild, C. (2007). [Liquid } \\
\text { Mind]. Relax: A Liquid } \\
\text { Mind Experience. } \\
\text { Sausalito, CA: Real Music } \\
\text { (2007). }\end{array}$ & Ages 8-12 & Relaxing/Sedative & $\begin{array}{l}\text {-Slow steady tones to promote rest and deep } \\
\text { breathing. } \\
\text {-Electronically produced music; reduces possible } \\
\text { negative associations with other forms of "relaxing" } \\
\text { music. } \\
\text {-Minimal changes in tonality and volume. }\end{array}$ \\
\hline
\end{tabular}


place, as well as various musical elements described in Table 1. Music was also screened for lyric appropriateness both in language and subject matter (therefore Radio Disney songs were used instead of pop songs). Use of a limited number of CDs was intended to decrease the amount of variance in musical stimuli, while the CDs selected offered a range of very different options; lullabies, relaxing classical music, electronically produced relaxing music, upbeat earlychildhood songs, upbeat and fully orchestrated movie tunes, and pop/rock music with electronic instrumentation. While this intervention did not fully employ patient preferred music because patient and family preferences may have fallen outside the range of selections offered, the patient and family were instead offered the opportunity to choose recorded music from this preselected array. The limited range of CDs was intended to reduce variability in musical stimuli offered as well as to account for the limited time available to present and provide musical selections to patient/families. Music was selected based on titles popular amongst the inpatient population at the same hospital during the time of the study. There was no contact with the participants prior to the study so it was not possible to do a survey of music selection prior to the intervention. CDs were selected for the various Musical and therapeutic qualities described in Table 1 and to provide a range of musical styles and instrumentation. While stimulative CDs for ages 4-7 and 8-12 both had "Disney" in the title, "Disney's Greatest Hits" included songs from popular Disney movies and also served as an example of upbeat music with a full orchestral, or more complex acoustic instrumental accompaniment, while "Radio Disney Greatest Hits" was comprised of songs from both Disney live action movies and songs with frequent air play on popular radio at the time (example Taylor Swift, Jonas Brothers, Miley Cyrus). These songs served as an example of pop/rock style music with more electronic or modern instrumentation. Radio Disney was also selected as it did not contain content or language that might be objectionable to families participating in the study. The MT-BC instructed families to consider sedative CDs as a method for encouraging relaxation and easing sedation, and to consider engaging CDs as a method for encouraging anxiety reducing play prior to the IV start and alternate engagement during. Patients and families were first presented with the selections matching their child's chronological age but were allowed to select any CD from the collection. Music was played free field via a Sony ZS-Y3 Radio/CD player with volume levels marked for consistency. The CD player was placed on a shelf along the back wall of the treatment room consistently for all participants. The music therapist started the CD, checked for volume consistency at a central point in the exam room, and gave final instructions for effective use of recorded music prior to the imaging. These instructions included movement, singing, and game playing for patients/families who selected stimulative music and deep breathing, closing one's eyes, and rocking young children for those who selected relaxing music. The MT-BC was not present during IV catheter insertion or administration of sedation medications.

\subsection{CLI}

The CLI group was used as a comparison to determine if any other child life intervention would evoke results similar to the music interventions. Participants and families engaged in developmentally appropriate preparation for the IV start and MRI when developmentally appropriate facilitated by the same Certified Child Life Specialist (CCLS) for all the patients. Prior to the IV catheter insertion, children and families were engaged in pre-procedural play and education to decrease anxiety. When developmentally appropriate, medical play was used prior to IV insertion. In this medical play, the patient has the opportunity to place an IV catheter in a small cloth doll, in order to increase the child's understanding prior to IV insertion. Patients and family members were given the opportunity to select and practice play based coping mechanisms from a selection of toys and activities provided by the CCLS. These included bubble blowing, pinwheels, squeezing a stress ball, distraction with visually stimulating toys, play with cause and effect toys, books with visual puzzles and buttons which produced sound, and play with small digital games. Materials used were based on the patient's developmental level and preference. The CCLS gathered verbal statements from the patient and family and also assessed the patient's level of engagement with various materials to determine preference. During the IV start, the CCLS stayed with the child to encourage the use of these coping tools. Coping techniques could be modified in the moment due to the demands of the procedure and the engagement level of the patient. The CCLS remained with the family until the child was sedated.

\subsection{NI}

Participants received only parenteral sedation. 
The study was approved by the institutional human investigation committee and reviewed regularly by an independent Data and Safety Monitoring Board.

\section{OUTCOME MEASUREMENTS AND STATISTICAL ANALYSES}

\subsection{Data Analysis, Sample Size and Statistical Methodology}

All interventions were compared with the nonintervention (NI) group. All statistical procedures were performed using SPSS Version 19.0. Statistically significant differences were considered achieved at a p-value $\leq 0.05$.

\subsection{Outcome Measures}

The primary outcome was the need for repeat sedation medications in children undergoing sedation. In our experience, 1-12 year old children usually required greater than 2 sedation doses. Therefore, we hypothesized that a higher proportion of children in the study arm who received $\mathrm{NI}$ would require $>2$ sedation doses (needing severe sedation) in comparison to the children in the intervention arms.

\subsection{Statistical Analysis for the Primary Outcome and Power/Sample Size Calculation}

Our previous unpublished preliminary data had shown that repeated sedation doses (> 2 doses) was required by a lower proportion (approximately 20\%) of patients aged 12-18 years who received some form of Music Therapy in comparison to children who did not. We hypothesized that we would again obtain a $20 \%$ reduction in the proportion of patients requiring $>2$ doses of sedation who received either AMT or FML or $\mathrm{CLI}$ in comparison to patients who did not receive any intervention at all. The null hypothesis is that the proportions would be equal across all four study groups. Using a Fisher's Exact test with the criterion for significance (alpha) set at 0.05 , and selecting a 2-tailed test (which means that an effect in either direction will be interpreted) a sample size of 123 per group (total $\mathrm{n}=492$ ) would provide power of $80.2 \%$. This computation assumes that the difference in proportions $16 \%$ (20\% reduction from $80 \%$; specifically, $80 \%$ vs. $64 \%$ ). We randomized 471 children (well over the required $\mathrm{N}=152$ ) to the four arms of the study allowing us to conduct robust multiple planned secondary measures.

4.4. Statistical Analyses for Secondary Measures (comparisons between total sedation time, sedation success rates, adverse event rates, parental satisfaction, salivary stress biomarker levels and costs of procedure)

Parametric analysis of variance (ANOVA) models examined mean differences on variables continuously scaled between the four study arms. Scheffe post hoc tests were employed to balance Type I error rate and power, and as a control for the problem of multiple tests of hypotheses. If assumptions of normality and/or homogeneity of variance were violated, non-parametric

Table 2: Patient Demographics*

\begin{tabular}{|c|c|c|c|c|c|c|}
\hline Variable & Category & Active Music & $\begin{array}{l}\text { Facilitated Music } \\
\text { Listening }\end{array}$ & $\begin{array}{l}\text { Child Life } \\
\text { Intervention }\end{array}$ & $\begin{array}{c}\text { No } \\
\text { Intervention }\end{array}$ & Total \\
\hline N (Sample) & & 120 & 123 & 118 & 110 & 471 \\
\hline \multirow[t]{2}{*}{ Gender } & Male & $83(69.2 \%)$ & $75(61.0 \%)$ & $71(60.2 \%)$ & $60(54.5 \%)$ & $289(61.4 \%)$ \\
\hline & Female & 37 (30.8\%) & $48(39.0)$ & $47(39.8)$ & $50(45.5 \%)$ & $182(38.6 \%)$ \\
\hline \multirow[t]{2}{*}{ Ethnicity } & Caucasian & $62(51.7 \%)$ & $63(51.2 \%)$ & $62(52.5 \%)$ & $54(49.1 \%)$ & $241(51.2 \%)$ \\
\hline & African American & $45(37.5 \%)$ & 49 (39.8\%) & $44(37.3 \%)$ & $42(38.2 \%)$ & $180(38.2 \%)$ \\
\hline \multirow[t]{4}{*}{ Asian } & $3(2.5 \%)$ & $1(0.8 \%)$ & $0(0.0 \%)$ & $0(0.0 \%)$ & $4(0.8 \%)$ & \\
\hline & American Indian & $0(0.0 \%)$ & $1(0.8 \%)$ & $2(1.7 \%)$ & $0(0.0 \%)$ & $3(0.6 \%)$ \\
\hline & Mixed & $5(4.2 \%)$ & $7(5.7 \%)$ & $5(4.2 \%)$ & $8(7.3 \%)$ & 25 (5.3\%) \\
\hline & Not Documented & $5(4.2 \%)$ & $2(1.6 \%)$ & $5(4.2 \%)$ & $6(5.5 \%)$ & $18(3.8 \%)$ \\
\hline Age in years & & $4.93 \pm 3.02$ & $4.73 \pm 3.20$ & $4.25 \pm 2.89$ & $4.46 \pm 2.91$ & $4.60 \pm 3.01$ \\
\hline $\begin{array}{c}\text { Height in } \\
\text { centimeters }\end{array}$ & & $105.68 \pm 26.8$ & $101.60 \pm 33.1$ & $95.76 \pm 3.9$ & $103.40 \pm 28.3$ & $101.60 \pm 30.3$ \\
\hline $\begin{array}{l}\text { Weight in } \\
\text { Kilograms }\end{array}$ & & $21.89 \pm 10.31$ & $22.29 \pm 12.36$ & $20.44 \pm 10.33$ & $22.29 \pm 14.70$ & $21.73 \pm 11.99$ \\
\hline
\end{tabular}

*Data presented as mean/standard deviation or number/percentage within study arm. 
Kruskal Wallis tests were substituted, followed by Mann Whitney U pair-wise comparisons. Fisher's Exact test was employed for comparisons between two groups on categorically scaled variables.

\section{RESULTS}

\subsection{Study Participants and Demographics}

Overall, 471 participants (i.e. Table 2) were enrolled into four study arms; AMT ( $n=120)$, FML ( $n=123)$, CLI $(n=118)$, NI $(n=110) ; 381 / 471$ participants received an MRI of the brain, and $42 / 471$ of the spine. Demographics are presented below in Table 2.

\subsection{Primary Outcomes}

The majority of children in each of the four study arms required 2 or more doses of sedation medications (i.e. Table 3). Still, when examined using the Fisher's
Exact test, a higher percentage of those in the $\mathrm{NI}$ arm (75.5\%) required $>2$ doses in comparison to either AMT (64.2\%; $p=.09)$, FML $(67.5 \% ; p=.19)$, or CLI $(69.5 \% ; p=.37)$. All $p$-values are unadjusted for multiple comparisons against $\mathrm{NI}$.

Although an unplanned (post hoc) analysis, we further explored the proportional differences by type of tool intervention employed in the three intervention arms with the $\mathrm{NI}$ arm. For this comparison, in order to increase the degree of certainty for interpretation of significance, we selected only tools which had a sample size of 20 or greater (these eight tools are listed below in Table 4). The type of tool intervention employed within the three intervention arms was dependent upon a child's preference.

Although no statistically significant differences were found in the comparisons using the Fisher's Exact test,

Table 3: Sedation Doses Required by Study Arm for MRI Procedure*

\begin{tabular}{|c|c|c|c|c|c|}
\hline \multirow{2}{*}{ Sedation Doses } & \multicolumn{5}{|c|}{ Study Arm } \\
\hline & Active Music & FML & CLI & No Intervention & Total \\
\hline 1 Dose & $3(2.5 \%)$ & $4(3.3 \%)$ & $3(2.5 \%)$ & $2(1.8 \%)$ & $12(2.5 \%)$ \\
\hline 2 Doses & $40(33.3 \%)$ & $36(29.3 \%)$ & $33(28.0 \%)$ & $25(22.7 \%)$ & $134(28.5 \%)$ \\
\hline$>2$ Doses & 77 (64.2\%) & 83 (67.5\%) & 82 (69.5\%) & 83 (75.5\%) & 325 (69.0\%) \\
\hline Total & $120(100.0 \%)$ & $123(100.0 \%)$ & $118(100.0 \%)$ & $110(100.0 \%)$ & $471(100.0)$ \\
\hline
\end{tabular}

*Percent within Study Arm.

Table 4: Percent Need for Sedation > 2 Doses by Select Intervention Tool (Sample Sizes $\geq 20$ )

\begin{tabular}{|c|c|c|c|c|c|}
\hline TOOL & $\mathbf{N}$ & $\%$ & $\%$ difference & $\begin{array}{l}\text { Lower \% for Tool than } \\
\text { No Intervention? }\end{array}$ & $p$-value \\
\hline WSAS & 25 & $64.0 \%$ & \multirow{2}{*}{$11.5 \%$} & \multirow{2}{*}{ Yes } & \multirow{2}{*}{0.32} \\
\hline No Intervention & 110 & $75.5 \%$ & & & \\
\hline Radio Disney & 26 & $57.7 \%$ & \multirow{2}{*}{$17.8 \%$} & \multirow{2}{*}{ Yes } & \multirow{2}{*}{0.09} \\
\hline No intervention & 110 & $75.5 \%$ & & & \\
\hline Disney's Greatest Hits & 32 & $71.9 \%$ & \multirow{2}{*}{$3.6 \%$} & \multirow{2}{*}{ Yes } & \multirow{2}{*}{0.65} \\
\hline No Intervention & 110 & $75.5 \%$ & & & \\
\hline Ocean Drum & 36 & $75.0 \%$ & \multirow{2}{*}{$0.5 \%$} & \multirow{2}{*}{ Yes } & \multirow{2}{*}{1.00} \\
\hline No Intervention & 110 & $75.5 \%$ & & & \\
\hline Musical Books & 24 & $62.5 \%$ & \multirow{2}{*}{$13.0 \%$} & \multirow{2}{*}{ Yes } & \multirow{2}{*}{0.21} \\
\hline No Intervention & 110 & $75.5 \%$ & & & \\
\hline Bubbles & 31 & $64.5 \%$ & \multirow{2}{*}{$11.0 \%$} & \multirow{2}{*}{ Yes } & \multirow{2}{*}{0.26} \\
\hline No Intervention & 110 & $75.5 \%$ & & & \\
\hline Just “books” & 20 & $80.0 \%$ & \multirow{2}{*}{$4.5 \%$} & \multirow{2}{*}{ No } & \multirow{2}{*}{0.78} \\
\hline No Intervention & 110 & $75.5 \%$ & & & \\
\hline
\end{tabular}

*Statistically significant at $\leq 0.05$. 
the trend reveals a clinically significant pattern. The largest proportional differences in not needing greater than 2 doses were found in the guitar $(18.4 \% ; p=0.03)$ and the Radio Disney (17.8\%; $p=0.09)$, followed by Musical Books (13.0\%; $\mathrm{p}=0.21)$, Wee Sing Animal Songs (WSAS) $(11.5 \%)$, or bubbles intervention $(11.0 \%)$.

\subsection{Secondary Outcomes}

\subsubsection{Total Sedation Time}

Output from the ANOVA procedure revealed that there were no statistically significant pair-wise mean differences among the four intervention groups for TST (i.e. Table 5), though FML showed a slightly decreased TST. Again, upon another unplanned (post hoc) analysis within the FML group, we observed interesting differences between the types of music used and mean total sedation time. Children under the age of 2 years who listened to Wee Sing Animal Songs (WSAS: $\mathrm{n}=20$ ) had significantly lower TST than children of the same age group who received no intervention $(\mathrm{NI}: \mathrm{n}=35$ ), $(p=0.001)$. To ensure that this effect was not simply due to listening to music in that age group, we compared, using the Mann Whitney U procedure, TST among children under 2 years who listened to Slumber Babies Lullabies (SBL: $n=16)$ ) with the similarly aged $\mathrm{NI}$ group and found no significant differences. The total sedation drug doses by weight in the WSAS group trended towards significance $(p<0.062)$ in being lower than doses used in NI or SBL.

\subsubsection{Sedation Success Rates}

All participants completed the procedure. Prior to the MRI procedure $97 \%-99 \%$ of all participants across study arms were classified as in deep sedation with no response to voice commands.

\subsubsection{Adverse Events Rates}

There were no statistically significant differences in adverse events among the study groups. Minor adverse events occurred in $24.8 \%$ (117/471) of participants. The highest percentage occurred in AMT (28.3\%). The most commonly occurring adverse event in all arms was desaturation, with the highest percentage $(25.8 \%)$ occurring in AMT. Overall, oxygen supplementation was the most frequently (30.6\%) performed intervention. Airway maneuvers were performed on 24 participants and 4 participants needed caffeine as a reversal agent, 2 of them in NI. Six

Table 5: Mean Total Sedation Time Across Study Arm

\begin{tabular}{|c|c|c|c|c|c|c|c|c|}
\hline \multicolumn{4}{|c|}{ Time Variable Measured } & \multicolumn{3}{c|}{ 95\% Confidence Interval of Mean } \\
\hline $\begin{array}{c}\text { Total Sedation } \\
\text { Time }\end{array}$ & N & Mean & SD & Std. Err & LB & UB & Min & Max \\
\hline \hline Active Music & 120 & 131.78 & 42.63 & 3.89 & 124.07 & 139.48 & 72 & 312 \\
\hline FML & 123 & 122.97 & 40.69 & 3.67 & 115.71 & 130.23 & 54 & 285 \\
\hline CLI & 118 & 134.95 & 61.29 & 5.64 & 123.78 & 146.12 & 56 & 524 \\
\hline No Intervention & 110 & 135.21 & 38.77 & 3.70 & 127.88 & 142.54 & 54 & 250 \\
\hline Total & 471 & 131.07 & 46.89 & 2.16 & 126.83 & 135.32 & 54 & 524 \\
\hline
\end{tabular}

N: Sample size; SD: Standard Deviation; Std.Err: Standard Error; LB: Lower Bound; UB: Upper Bound; Min: Minimum Value; Max: Maximum Value.

Table 6: Parents Perception of Intervention Type Employed ${ }^{\star}$

\begin{tabular}{|c|c|c|c|c|c|}
\hline \multirow{2}{*}{ Perception } & \multicolumn{4}{|c|}{ Study Arm } \\
\cline { 2 - 6 } & Active Music & FML & CLI & No Intervention \\
\hline \hline Not documented & $1(0.8 \%)$ & $1(0.8 \%)$ & $0(0.0 \%)$ & $0(0.0 \%)$ & $2(0.4 \%)$ \\
\hline Strongly Disliked & $0(0.0 \%)$ & $1(0.8 \%)$ & $0(0.0 \%)$ & $2(1.8 \%)$ & $3(0.6 \%)$ \\
\hline Disliked & $0(0.0 \%)$ & $3(2.4 \%)$ & $0(0.0 \%)$ & $2(1.8 \%)$ & $5(1.1 \%)$ \\
\hline Neutral & $6(5.0 \%)$ & $13(10.6 \%)$ & $1(0.8 \%)$ & $51(46.4 \%)$ & $71(15.1 \%)$ \\
\hline Liked & $22(18.3 \%)$ & $39(31.7 \%)$ & $20(16.9 \%)$ & $10(9.1 \%)$ & $91(19.3 \%)$ \\
\hline Strongly Liked & $91(75.8 \%)$ & $66(53.7 \%)$ & $97(82.2 \%)$ & $45(40.9 \%)$ & $299(63.5 \%)$ \\
\hline Total & $120(100.0 \%)$ & $123(100.0 \%)$ & $118(100.0 \%)$ & $110(100.0 \%)$ & $471(100.0 \%)$ \\
\hline
\end{tabular}

${ }^{*}$ Percent within Study Arm. 
patients ( 2 in each intervention arm) required additional observation in the emergency department. Two patients, both in $\mathrm{NI}$, required hospitalization for further monitoring and were then discharged within 24 hours.

\subsubsection{Parental Satisfaction}

As shown below in Table 6, using a cross tabulation procedure, more parents in all three intervention arms strongly liked the intervention as compared to NI. It is interesting to note that $\mathrm{CLI}$ received the highest percentage of "strongly liked". It is noteworthy that parental satisfaction does not necessarily match effectiveness of our outcome measures.

\subsubsection{Salivary Cortisol and Pro-Inflammatory Cytokine Levels}

We measured the levels of the stress hormone cortisol as well as levels of some inflammatory cytokines in the saliva of the patients pre- and postsedation. The changes of these biomarker levels were expressed as ratios of post/pre-sedation levels. Based upon ANOVA output, no significant differences were observed in the ratios of post/pre-sedation levels of any of the intervention arms for any of the biomarkers (cortisol, MCP-1, IL-1 alpha, IL-1 beta, IL-6, IL-8 or TNF-alpha) tested as compared with $\mathrm{NI}$ (data not shown). The WSAS group demonstrated statistically significant differences between the post/pre-sedation ratios of salivary MCP-1 $(p<.034)$ as compared with the $\mathrm{NI}$ group and the SBL group of the FML arm of children of the same age.

\subsubsection{Costs of Procedure}

Our institutional administrative mean charges in the sedation suite are approximately $\$ 3.59$ per minute, including the cost of drugs, personnel time and suite time. Based on the outcome from the Mann Whitney $U$ test, the mean savings garnered by the decreased TST in the WSAS FML group were notable than in children in the $\mathrm{NI}$ group of the same age range. Also, mean costs in the $\mathrm{NI}$ group were higher $\$ 485.40 \pm 139.19$ in comparison to any of the intervention arms, especially the FML arm, with mean costs of $\$ 441.45 \pm 146.06$.

\section{DISCUSSION}

Different forms of Music Therapy have been used in clinical practices. However, literature regarding the research in applying MT in clinical practice and its immunological effects is generally sparse. Indeed, experts have suggested that results from current research on the use of MT for pediatric pre-procedural sedation and amelioration of pain/stress/anxiety, cannot be translated into clinical practice because of substantial heterogeneity among studies that have used different definitions, inconsistent outcomes and less robust study designs $[17,21,23]$. A CLI intervention group was used as a comparison group to ensure that any child life intervention technique did not impact the parameters we were studying, and that changes we saw were indeed specific to Music Therapy interventions.

The results of our large prospective four-arm clinical study comparing AMT, FML and CLI with NI on children 12 years and younger who were scheduled to receive parenteral sedation for MRI studies revealed that a lower proportion of children who received some form of intervention (either AMT, FML, or CLI) needed $>2$ doses of sedation in comparison to those who did not have an intervention at all. The lowest proportion of those needing $>2$ doses of sedation had received AMT. It is important to note, however, that we had powered based upon an effect size difference of $16 \%(80 \%$ vs. $64 \%)$. The percentages actually found were higher than we expected $(>60 \%)$ in the intervention groups (AMT: 64.1\%, FML: $67.5 \%$, CLI: $69.5 \%$ ), and lower than expected $(<80 \%)$ in the no intervention group $(75.5 \%)$. Further, no significant differences were found between the interventions in total dose sedation medications, total sedation time, and levels of salivary cortisol and cytokines. However, use of Radio Disney in FML, did show decrease in the use of sedation medication, but no other measured parameter. We are planning to address this finding in more detail in future studies.

A planned sub-analyses revealed that children two years and younger, who engaged in FML involving upbeat and familiar music, had a lower total sedation time compared to those who did not get any intervention, suggesting a potential mechanism for conducting cost-effectiveness analysis. Our current controlled study with a large number of pediatric patients demonstrates that certain forms of FML are indeed beneficial to patients undergoing sedation, especially in terms of reduced Total Sedation Time and the lowered associated hospital costs. The mean difference of $\$ 44.40$ resulting from the reduced hospital stay of patients in the FML arm as compared with the $\mathrm{NI}$ arm, multiplied by the annual number of MRI scans performed would result in substantial net savings yearly. These cost savings demonstrate the importance of implementing such non-invasive, inexpensive interventions such as age-appropriate FML routinely. 
The significant differences found in the post/pre ratios for MCP-1 and IL-8 are interesting although challenging to interpret. MCP-1 is a pro-inflammatory marker best known for its ability to recruit monocytes to sites of damage or infection [24]. IL-8, also proinflammatory, is principally known to be a recruiter and activator of neutrophils [25]. While MCP-1 has been demonstrated to increase the permeability of the bloodbrain barrier in mice, the time-frame important for sedation was not directly investigated in those studies [26-28]. This, however, does not preclude a potential use for MCP-1 as a biomarker of intervention efficacy in future studies in this age group. It is noteworthy that not all interventions, indeed not even all forms of FML, resulted in lowered TST or MCP-1 changes in our study. This is consistent with previous studies in adults demonstrating the "Mozart effect" i.e. that exposure to certain Mozart sonatas, but not other similar classical music forms, improved outcomes in several disorders [29]. It is possible that WSAS presented a better "fit" for the two-year-olds in the study than any of the other types of music presented to the other age groups. The role of a Music Therapist is therefore important in designing MT interventions since Music Therapists are trained to consider all factors impacting preferences, how those preferences may change over time and how preferences impact how one perceives an intervention. Musical preferences are highly individual and specific [30], and perhaps the music presented to this group by the Music Therapist was the closest match to their preferred music. It is therefore important to examine in greater detail the types of music exposures that produce favorable outcomes, as did WSAS in our patients aged 2 years and younger. Similarly, it is important to identify other therapeutic modalities that improve not just TST but also increase MCP-1 levels, and to study the biology of the mechanisms associated with these changes.

The inclusion of Wee Sing Animal Songs as a listening selection for FML was designed to promote engagement and distraction in children under the age of two. This was of primary importance during the IV start. Although no significant changes in observed distress were noted amongst young patients who participated in FML, the decreased time in sedation and reduction in sedation medication may be an indication that the inclusion of upbeat engaging music did reduce patient anxiety during the IV start or promote calming following the IV start. This reduction of anxiety prior to the administration of sedation medication could result in faster sedation times and the use of less medication. Additionally, the inclusion of familiar sound stimuli during sedation may have reduced patient's anxiety related to physiological sensation associated with sedation medication. Young children's attention to the musical stimuli may have reduced their perception of dizziness or sleepiness association with the medication and decreased their tendency to fight sleep. Similar stimuli are becoming more common in the daily care of young children as recorded music, musical toys, interactive technology based toys and television are increasingly used as entertainment. The inclusion of this familiar stimulus may over-ride some of the unknown and intimidating aspects of the hospital environment, especially those associated with medical procedures and sedation.

Parental satisfaction was highest with CLI and neutral with FML; this along with high parental perceptions suggests that parents prefer a high level of engagement of active therapists with their children during these procedures. We will examine these findings in greater detail in collaboration with experts in this area in future studies. We will also study the implications of our overall findings for clinical practices, since the importance of engaging Child Life and Music Therapy professionals in these interventions appears to be important in increasing parental satisfaction and in decreasing sedation medication doses in children, thus making the experience in the clinical setting more comfortable for all involved. Since our study showed that "Wee Sing Animal Songs" resulted in significant decrease in total sedation time and reduction of associated costs, we wish to recommend its use (as well as other similar modalities to be identified in future studies) in clinical practice for age appropriate patients who require procedural sedation. In addition since more parents in all three intervention arms strongly liked the intervention as compared to no intervention, clinical practices should be strongly encouraged to integrate music therapy and child life interventions as a routine part of the standard of care for children undergoing procedural sedation. In addition, these interventions might prove to be useful in other pediatric clinical settings to mitigate patient discomforts.

This study had some limitations. The study could not be blinded for obvious reasons. To address this limitation, we chose multiple measures that were objective as the primary and secondary outcomes. The medications used at our institution for MRI procedural sedations are less commonly used at other institutions and this may limit the generalizability of the study. However, this limitation is less likely to be of 
significance because every patient who participated in the study received sedation medications by institutional protocol, thus maintaining consistency across the study arms.

\section{CONCLUSION}

Parental perception and satisfaction of use of Active Music Therapy, facilitated music listening and child life intervention was higher than no intervention in pediatric sedation for MRI. Further, a true reduction in total sedation time in the very young age group who were exposed to music involving upbeat and engaging music suggests an area of opportunity to conduct costeffectiveness analysis. The role of a Music Therapist in clinical practice is therefore important in designing MT interventions since Music Therapists are trained to consider all factors impacting preferences, how those preferences may change over time and how preferences impact how one perceives an intervention.

\section{ACKNOWLEDGEMENTS}

We acknowledge the expertise of child life specialists Karin Angelos and Salika Coleman, the cooperation of the staff in the sedation and MRI suites of the Children's Hospital of Michigan, as well as the technical expertise of Kirk Trentham in conducting this study.

\section{CLINICAL TRIALS}

This study is registered at www.clinicaltrials.gov; study ID\#: RC1AT005699

\section{FUNDING SOURCE}

All phases of this study were supported by an NIH grant, RC1AT005699

\section{FINANCIAL DISCLOSURE}

None of the authors have financial relationships relevant to this article to disclose

\section{CONFLICT OF INTEREST}

None of the authors have conflicts of interest to disclose

\section{ABBREVIATIONS}
AMT = Active Music Therapy
CAM = Complementary and Alternative Medicine
CCLS $=$ Certified Child Life Specialist

CLI = Child Life Intervention

DSMB = Data Safety and Monitoring Board

DT = Distraction Therapy

FML $=$ Facilitated Music Listening

$\mathrm{IL}-8=$ Interleukin-8

MCP-1 = Monocyte Chemoattractant Protein-1

$\mathrm{MRI}=$ Magnetic Resonance Imaging

MT-BC = Board Certified Music Therapist

$\mathrm{NI}=$ No Intervention

$\mathrm{SBL} \quad=$ Slumber Babies Lullabies

TST $=$ Total Sedation Time

WSAS $=$ Wee Sing Animal Songs

\section{REFERENCES}

[1] Cravero JP, Blike GT. Review of pediatric sedation. Anaesth Analg 2004; 99: 1355-64. http://dx.doi.org/10.1213/01.ANE.0000134810.60270.E8

[2] Daud YN, Carlson DW. Pediatric sedation. Pediatr Clin North Am 2014; 61: 703-17. http://dx.doi.org/10.1016/i.pcl.2014.05.003

[3] Langhan ML, Shabanova V, Li FY, Bernstein SL, Shapiro ED. A randomized controlled trial of capnography during sedation in a pediatric emergency setting. Am J Emerg Med 2015; 33: 25-30.

http://dx.doi.org/10.1016/j.ajem.2014.09.050

[4] Emrath ET, Stockwell JA, McCracken CE, Simon HK, Kamat PP. Provision of deep procedural sedation by a pediatric sedation team at a freestanding imaging center. Pediatr Radiol 2014; 44: 1020-5. http://dx.doi.org/10.1007/s00247-014-2942-z

[5] Caperell K, Pitetti R. Is higher ASA class associated with an increased incidence of adverse events during procedural sedation in a pediatric emergency department? Pediatr Emerg Care 2009; 25: 661-4. http://dx.doi.org/10.1097/PEC.0b013e3181bec7cc

[6] McCaffrey R, Locsin RC. Music listening as a nursing intervention: A symphony of practice. Holist Nurs Pract 2002; 16: 70-7. http://dx.doi.org/10.1097/00004650-200204000-00012

[7] Avers L, Mathur A, Kamat D. Music therapy in pediatrics. Clin Pediatr 2007; 46(7): 575-57. http://dx.doi.org/10.1177/0009922806294846

[8] Ghetti CM. Music therapy as procedural support for invasive medical procedures: toward the development of music therapy theory. Nord J Music Ther 2012; 21: 3-25. http://dx.doi.org/10.1080/08098131.2011.571278

[9] Stouffer JW, Shirk BJ, Polomano RC. Practice guidelines for music interventions with hospitalized pediatric patients. J Pediatr Nurs 2007; 22: 448-56. http://dx.doi.org/10.1016/j.pedn.2007.04.011

[10] Bishop B, Christenberry A, Robb S, Toombs Rudenberg M. Music therapy and child life interventions with pediatric burn patients. In: Froelich MA, editor. Music Therapy with Hospitalized Children: A Creative Arts Child Life Approach. Cherry Hill: Jeffry Books 1996; pp. 87-108. 
[11] Loewy JV, MacGregor B, Richards K, Rodreguez J. Music therapy in pediatric pain management: Assessing and attending to the sounds of hurt, fear and anxiety. In: Loewy JV, editor. Music Therapy in Pediatric Pain. Cherry Hill: Jeffrey Books 1997; pp. 45-46.

[12] Walworth DD. Procedural-support music therapy in the healthcare setting: A cost-effectiveness analysis. J Pediatr Nurs 2005; 20: 276-84. http://dx.doi.org/10.1016/j.pedn.2005.02.016

[13] Myskja A, Lindbaek M. How does music affect the human body? Tidsskr Nor Laegeforen 2000; 120: 1182-5.

[14] Lane D. Effects of music therapy on immune function of hospitalized patients. Qual Life 1994; 3: 74-80.

[15] Evans D. The effectiveness of music as an intervention for hospital patients: A systematic review. J Adv Nurs 2003; 37: 8-18. http://dx.doi.org/10.1046/j.1365-2648.2002.02052.x

[16] Kirby LA, Olivia R, Sahler OJZ. Music therapy and pain management in pediatric patients undergoing painful procedures: A review of the literature and a call for research. J Altern Med Res 2010; 2: 7-16.

[17] Yip P, Middleton P, Cyna AM, Carlyle AV. Nonpharmacological interventions for assisting the induction of anaesthesia in children. Cochrane Database Syst Rev 2009; 3: 1-62. http://dx.doi.org/10.1002/14651858.cd006447.pub2

[18] Standley JM, Whipple J. Music therapy with pediatric patients: A meta-analysis. In: Robb SL, editor. Music Therapy in Pediatric Healthcare: Research and Evidence Based Practice. Silver Spring: American Music Therapy Association Inc 2003; pp. 1-18.

[19] Grasso MC, Button BM, Allison DJ, Sawyer SM. Benefits of music therapy as an adjunct to chest physiotherapy in infants and toddlers with cystic fibrosis. Pediatr Pulmonol 2000; 29: 371-81.

http://dx.doi.org/10.1002/(SICI)10990496(200005)29:5<371::AID-PPUL6>3.0.CO;2-K

[20] Lai HL, Liao KW, Huang CY, Chen PW, Peng TC. Effects of music on immunity and physiological responses in healthcare workers: A randomized controlled trial. Stress Health 2013; 29: $91-8$.

http://dx.doi.org/10.1002/smi.2429
[21] Klassen JA, Liang Y, Tjosvold L, Klassen TP, Hartling L. Music for pain and anxiety in children undergoing medical procedures: A systematic review of randomized controlled trials. Ambul Pediatr 2008; 8: 117-28.

http://dx.doi.org/10.1016/j.ambp.2007.12.005

[22] Loewy J, Hallan C, Friedman E, Martinez C. Sleep/sedation in children undergoing EEG testing: a comparison of chloral hydrate and music therapy. J Perianesth Nurs 2005; 20: 32332.

\section{http://dx.doi.org/10.1016/j.jopan.2005.08.001}

[23] Edwards J. Possibilities and problems for evidence-based practice in music therapy. Arts Psychother 2005; 32: 293301.

http://dx.doi.org/10.1016/.j.aip.2005.04.004

[24] Yadav A, Saini V, Arora S. MCP-1: chemoattractant with a role beyond immunity: A review. Clin Chim Acta 2010; 411: 1570-9. http://dx.doi.org/10.1016/..cca.2010.07.006

[25] Semple BD, Kossmann T, Morganti-Kossmann MC. Role of chemokines in CNS health and pathology: A focus on the CCL2/CCR2 and CXCL8/CXCR2 networks. J Cereb Blood Flow Metab 2010; 30: 459-73. http://dx.doi.org/10.1038/jcbfm.2009.240

[26] Stamatovic SM, Shakui P, Keep RF, et al. Monocyte chemoattractant protein-1 regulation of blood-brain barrier permeability. J Cereb Blood Flow Metab 2005; 25: 593-606. http://dx.doi.org/10.1038/sj.jcbfm.9600055

[27] Stamatovic SM, Keep RF, Kunkel SL, Andjelkovic AV. Potential role of MCP-1 in endothelial cell tight junction 'opening': signaling via Rho and Rho kinase. J Cell Sci 2003; 116: 4615-28. http://dx.doi.org/10.1242/jcs.00755

[28] Gröschl M. The physiological role of hormones in saliva. Bioessays 2009; 31: 843-52. http://dx.doi.org/10.1002/bies.200900013

[29] Rauscher FH, Shaw GL, Ky KN. Music and spatial task performance. Nature 1993; 365: 611. http://dx.doi.org/10.1038/365611a0

[30] Standley JM. Music research in medical treatment. In: Smith D, Ed. Effectiveness of Music Therapy Procedures: Documentation of Research and Clinical Practice. Silver Spring: American Music Therapy Association 2000; pp. 1-64.

http://dx.doi.org/10.6000/1929-4247.2016.05.02.3

(C) 2016 Mathur et al.; Licensee Lifescience Global.

This is an open access article licensed under the terms of the Creative Commons Attribution Non-Commercial License (http://creativecommons.org/licenses/by-nc/3.0/) which permits unrestricted, non-commercial use, distribution and reproduction in any medium, provided the work is properly cited. 